\title{
Left Ventricular Remodeling in Patients with Primary Percutaneous Coronary Intervention for Anterior Myocardial Infarction
}

\author{
Al-Shimaa M. Sabry, Khaled E. El-Rabat, Ali I. Attia, Hager I. Abd El-Fatah
}

\begin{abstract}
:
Department of cardiology, Benha Objectives: Evaluation of the role of speckle tracking faculty of medicine, Benha University, Egypt

Correspondence to: Al-Shimaa M. Sabry, department of cardiology, Benha faculty of medicine, Benha University, Egypt echocardiography in predicting left ventricular remodeling after anterior ST elevation myocardial infarction (STEMI) and successful primary percutaneous coronary intervention (PCI). Methods: A total of 100 first anterior STEMI and successful primary PCI were evaluated and divided into two groups according to the occurrence Email:

shimaa.sabry@fmed.bu.edu.eg

Available online: 13 September, 2020 of left ventricular (LV) remodeling; remodeling group $(n=26)$ and non remodeling group $(n=74)$. Conventional and speckle tracking echocardiography were performed within 3 days of admission and 3 months later. Results: Twenty-six (26\%) patients had LV remodeling at 3-month follow-up. They had comparable baseline clinical and echocardiographic characteristics with the non remodeling group except for $\beta$-blockers use $(\mathrm{P}=0.043)$, lower LV global longitudinal (LVGLS) and circumferential (LVGCS) strain ( $\mathrm{P}<0.001)$. An adjusted multivariate logistic regression analysis revealed that baseline LVGLS to be the only significant independent predictor for occurrence of LV remodelling (hazard ratio $=1.68,95 \% \mathrm{CI}: 1.35-2.09, \mathrm{p}=0.001)$. ROC curve analysis showed that a cut-off baseline LVGLS value $<-9.0 \%$ and LVGCS $<-11.1$ predicted 3 months LV remodelling. Conclusion: Early LV global longitudinal and circumferential strain can predict LV remodeling in anterior STEMI.
\end{abstract}

Keywords: Left ventricular remodelling; myocardial infarction; global longitudinal strain. 


\section{Introduction}

The ongoing improvements in early diagnosis and successful invasive management of patients with acute myocardial infarction resulted in reduced mortality rates due to complication of acute myocardial infarction. However, even with successful percutaneous coronary intervention (PCI), the risk of left ventricular remodeling (LVR) could not be excluded (1).

Left ventricular remodeling is deleterious process that starts with the acute phase of myocardial infarction and leads to progressive LV dilatation with systolic dysfunction. LV remodeling is a predictor of heart failure and increased mortality following acute myocardial infarction (2). Postinfarct LV remodeling occurs in $30 \%$ patients with prior history of myocardial infarction (3).

Although, many prior studies had evaluated clinical and echocardiographic parameters predisposing to left ventricular remodeling after acute myocardial infarction, there are still gaps in our data, and some clinical factors or diagnostic measures failed to predict left ventricular remodeling. Therefore, the present study aimed to evaluate the value of global longitudinal strain measured by speckle tracking echocardiography (STE) in predicting left ventricular remodeling after acute anterior myocardial infarction treated with primary PCI.

\section{Patients and methods}

\section{Patients:}

This prospective study was conducted at the coronary care unit of Benha University Hospitals, Egypt and included 100 patients with the first acute anterior STEMI treated with primary PCI. According to the presence of left ventricular remodeling 3 months later, patients were divided into two groups: patient without LV remodeling (group I) and patient with LV remodeling (group II) , LV remodeling was defined as $\geq 20 \%$ increase in LV end-diastolic volume (LVEDV) and/or end-systolic volume (LVESV) at 3-month follow-up compared with the baseline examination (4).

Exclusion criteria were history of myocardial infarction or any other heart muscle disease with persistent wall motion abnormalities, any significant general disorder of potential influence on regional or global LV wall motion, significant arrhythmia (including atrial fibrillation and advanced extrasystolic arrhythmia), previous pacemaker or cardioverter-defibrillator implantation, uncompleted reperfusion therapy (coronary artery bypass grafting or repeated PCI), and very poor image quality.

The patients provided informed consent to participate in the study. This study was approved by the hospital's ethics committee. 


\section{Echocardiography:}

Transthoracic echocardiography was performed in the left lateral position using the commercially available ultrasound system (Philips EPIQ 7 Ultrasound System, Andover, MA, USA) equipped with $3.5-\mathrm{MHz}$ phased array transducer. Two-dimensional (2D) and speckle tracking echocardiography was done within $72 \mathrm{~h}$. of symptoms onset.

LV ejection fraction and LV volumes (enddiastolic and end-systolic) were calculated using the modified Simpson's method at baseline and after 3 months. The left ventricle was divided into 17 segments and a scoring system (1, normal; 2, hypokinesia; 3 , akinesia; 4 , dyskinesia) was used to calculate WMSI as follows; sum of the segment scores divided by the number of segments scored (5).
LV global longitudinal strain (LVGLS) was measured by two-dimensional (2D) speckle tracking echocardiography in the apical 4-, 2chamber and long-axis views using appropriate frame rates. LV circumferential strain was measured in the three short-axis views (basal, midventricular, and apical) (6).

The time interval between R-wave and aortic valve was automatically calculated and used as a reference for apical 4- and 2-chamber views. We used three points to define the mitral annulus and LV apex. Endocardial border was manually traced, and the width of the region of interest was adjusted to include the myocardial thickness. Then, the software automatically tracks and calculates LV strain (Figure 1).

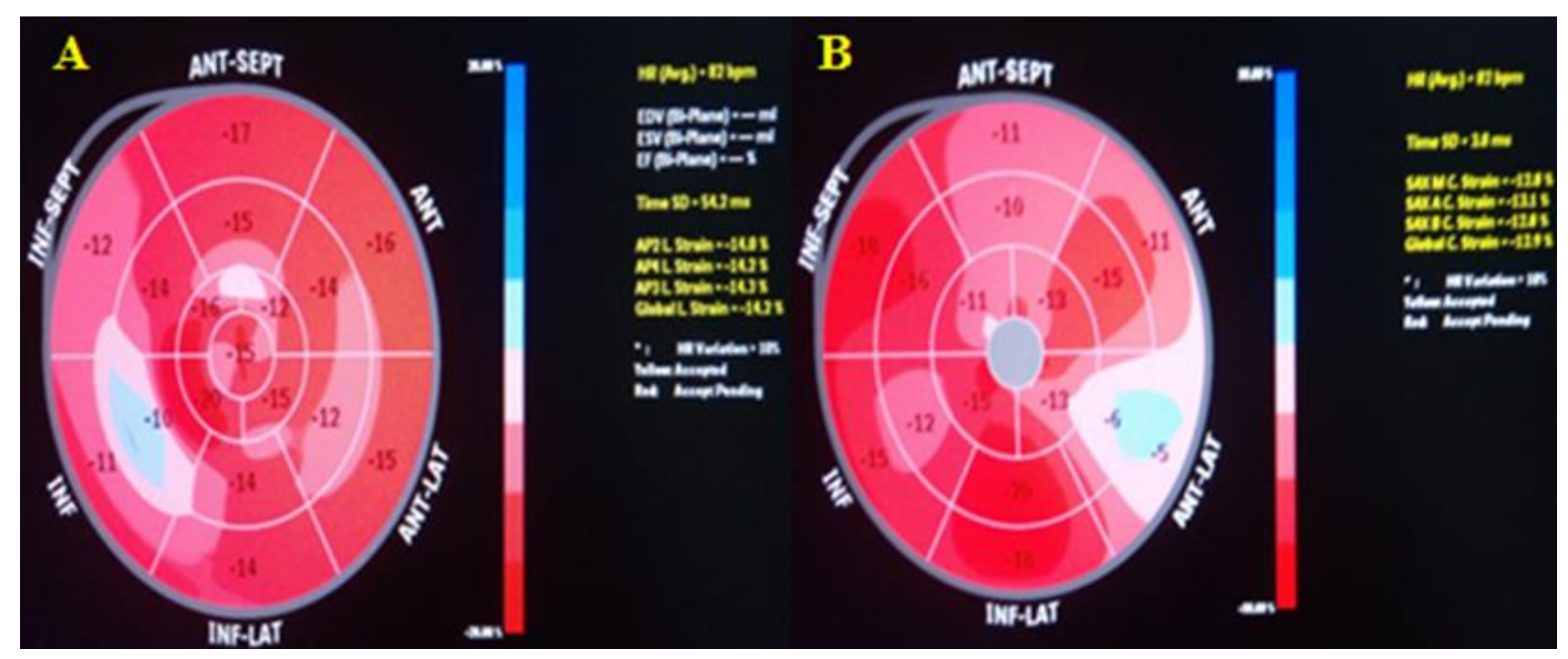

Figure 1: Baseline LV global longitudinal strain (LVGLS) and circumferential strain (LVGCS) of a patient from the remodeling group. A, bull's-eye LVGLS $=-14.2 \%$; and B, bull's-eye LVGCS $=-12.9 \%$. 
Statistical analysis: Data management and statistical analysis were performed using SPSS software v23 (IBM, Armonk, NY, USA). Numerical data was summarized as means and standard deviations.

Categorical data was summarized as numbers and percentages. Comparisons between nonremodeling and remodeling groups were performed using independent $t$ test for numerical variables and Chi-square test or Fisher exact test for categorical variables.

ROC analysis was done for using baseline GLS and GCS in predicting LV remodeling. Area under curve, best cutoff point and diagnostic indices including sensitivity and specificity were calculated. Logistic regression analysis was done for prediction of LV remodeling. Odds ratio with $95 \%$ confidence interval were calculated. All P values were two sided. P value less than 0.05 was considered significant.

\section{Results:}

\section{Baseline Characteristics:}

A total of 100 patients with anterior STEMI who underwent primary PCIs were included in our study. The mean age of the study population was $59 \pm 6$ years and $72(72 \%)$ of the patients were males. According to occurrence of LV remodeling after 3 months, the study population was divided into two groups: LV remodeling group =26 (26\%) patients and non remodeling group =74 (74\%) patients. Baseline demographics and angiographic characteristics of the study population are presented in Table 1. There was no significant statistical difference in baseline characteristics between the two groups. Patients without LV remodeling were more likely to receive beta blockers (68 patients "91.9\%" vs. 20 patients "76.9\%"; P = 0.043]. All patients of both groups received aspirin, clopidogrel and statin.

\section{Echocardiographic parameters:}

The echocardiographic data of the patients with and without LV remodeling are presented in Table 2. There was no significant statistical difference between the two groups regarding conventional echocardiographic parameters including LV volumes, LVEF, and WMSI. However, speckle tracking echocardiography revealed that patients with LV remodeling had lower LV global longitudinal (LVGLS) and circumferential (LVGCS) strain values (-7.97 \pm 3.85 vs. $-12.8 \pm 2.48 \%$ and $-11.76 \pm 6.87$ vs. $-18.35 \pm 2.94$ respectively, $\mathrm{P}<0.001)$. 
Table 1: Baseline characteristics of the study population

\begin{tabular}{|c|c|c|c|}
\hline & $\begin{array}{l}\text { Remodeling } \\
\mathbf{n}=\mathbf{2 6}\end{array}$ & $\begin{array}{l}\text { Non remodeling } \\
\mathrm{N}=74\end{array}$ & $P$ value \\
\hline Age, years, mean \pm SD & $57 \pm 6$ & $59 \pm 6$ & 0.132 \\
\hline Male gender, n (\%) & $22(84.6)$ & $50(67.6)$ & 0.096 \\
\hline DM & $8(30.8 \%)$ & $16(21.6 \%)$ & 0.347 \\
\hline HTN & $16(61.5 \%)$ & $51(68.9 \%)$ & 0.491 \\
\hline Smoking & $12(46.2 \%)$ & $39(52.7 \%)$ & 0.566 \\
\hline Dyslipidemia & $20(76.9 \%)$ & $55(74.3 \%)$ & 0.731 \\
\hline Family history of CAD & $3(11.5 \%)$ & $10(13.5 \%)$ & 0.232 \\
\hline $\mathrm{SBP}(\mathrm{mmHg})$ & $122 \pm 21$ & $123 \pm 19$ & 0.779 \\
\hline DBP (mmHg) & $79 \pm 15$ & $81 \pm 14$ & 0.604 \\
\hline \multicolumn{4}{|l|}{ Killip class } \\
\hline $\mathbf{I}$ & $17(65.4)$ & $60(81.1)$ & 0.278 \\
\hline II & $3(11.5)$ & $4(5.4)$ & \\
\hline III & $2(7.7)$ & $2(2.7)$ & \\
\hline IV & $4(15.4)$ & $8(10.8)$ & \\
\hline \multicolumn{4}{|c|}{ Number of affected vessels } \\
\hline Single vessel & $5(19.2 \%)$ & $27(36.5 \%)$ & 0.129 \\
\hline Two vessel & $13(50 \%)$ & $22(29.7)$ & \\
\hline Multi-vessel & $8(30.8 \%)$ & $25(33.8 \%)$ & \\
\hline \multicolumn{4}{|l|}{ Medications } \\
\hline B-blockers & $20(76.9 \%)$ & $68(91.9 \%)$ & 0.043 \\
\hline ACEI/ ARBs & $24(92.3 \%)$ & $71(95.9 \%)$ & 0.603 \\
\hline Aspirin & $26(100 \%)$ & $74(100 \%)$ & - \\
\hline Clopidogrel & $26(100 \%)$ & $74(100 \%)$ & - \\
\hline Statin & $26(100 \%)$ & $74(100 \%)$ & - \\
\hline
\end{tabular}

DM = diabetes mellitus; HTN = hypertension; $\mathbf{C A D}=$ coronary artery disease; $\mathbf{S D}=$ standard deviation; $\mathbf{S B P}=$ systolic blood pressure; $\mathbf{D B P}=$ diastolic blood pressure; $\mathbf{b p m}=$ beat per minute; $\mathbf{A C E I}=$ angiotensin converting enzyme inhibitor; $\mathbf{A R B s}=$ angiotensin receptor blockers.

Table 2: Echocardiographic parameters of the study population

\begin{tabular}{lccc}
\hline & $\begin{array}{c}\text { Remodeling } \\
\mathbf{n = 2 6}\end{array}$ & $\begin{array}{c}\text { Non remodeling } \\
\mathbf{n = 7 4}\end{array}$ & P value \\
\hline LVEDV, ml & $118 \pm 15$ & $117 \pm 10$ & 0.66 \\
LVESV, ml & $57.1 \pm 9$ & $56 \pm 6.3$ & 0.479 \\
LVEF, \% & $52.9 \pm 3.19$ & $52.7 \pm 4.19$ & 0.692 \\
WMSI & $1.33 \pm 0.13$ & $1.32 \pm 0.14$ & 0.844 \\
E(m/sec) & $63.9 \pm 17.4$ & $57.7 \pm 18$ & 0.128 \\
A(m/sec) & $66.7 \pm 5.5$ & $68.9 \pm 6$ & 0.107 \\
E/A ratio & $0.8 \pm 0.3$ & $1 \pm 0.3$ & 0.075 \\
E/ e' & $10.5 \pm 3.7$ & $9.3 \pm 3.5$ & 0.149 \\
LVGLS \% & $-7.97 \pm 3.85$ & $-12.8 \pm 2.48$ & $<0.001$ \\
LVGCS \% & $-11.76 \pm 6.87$ & $-18.35 \pm 2.94$ & $<0.001$ \\
\hline
\end{tabular}

LVEDV = left ventricular end diastolic volume; LVESV = left ventricular end systolic volume; $\mathbf{L V E F}=$ left ventricular ejection fraction; WMSI= wall motion score index; LVGLS = left ventricular global longitudinal strain; LVGCS = left ventricular global circumferential strain. 


\section{Prediction of left ventricular remodeling:}

Univariate analysis revealed that the use of $\beta$ blockers (BB), LV global longitudinal strain (LVGLS) and LV global circumferential strain (LVGCS) were significantly associated with LV remodeling $(p<0.05)$. Multivariate analysis using the forward stepwise method revealed that LVGLS was the only independent predictor of $\mathrm{LV}$ remodeling in patients with acute anterior myocardial infarction treated with primary PCI (hazard ratio $=1.68,95 \% \mathrm{CI}: 1.35-2.09, \mathrm{p}=0.001)$. For each one unit decrease in LVGLS, risk of remodeling increase by $68 \%$.

ROC curve was used to test the diagnostic value (overall accuracy) of LV global longitudinal strain (LVGLS) and circumferential strain (LVGCS) in predicting LV remodeling in patients with acute anterior myocardial infarction treated with primary PCI. LVGLS cutoff value of $<-9.0$ (sensitivity $=77 \%$, specificity $=93.2 \%$, AUC 0.85, 95\% CIs $0.736-0.963, \mathrm{P}<0.001)$ and LVGCS cutoff value of $<-11.1$ (sensitivity $=69 \%$, specificity $=100 \%$, AUC $0.75,95 \%$ CIs 0.595 $-0.908, \mathrm{P}<0.001)$ had the best diagnostic accuracy in predicting LV remodeling (Figure 2). AUC of LVGLS was higher than that of LVGCS, so GLS was more accurate in predicting LV remodeling.

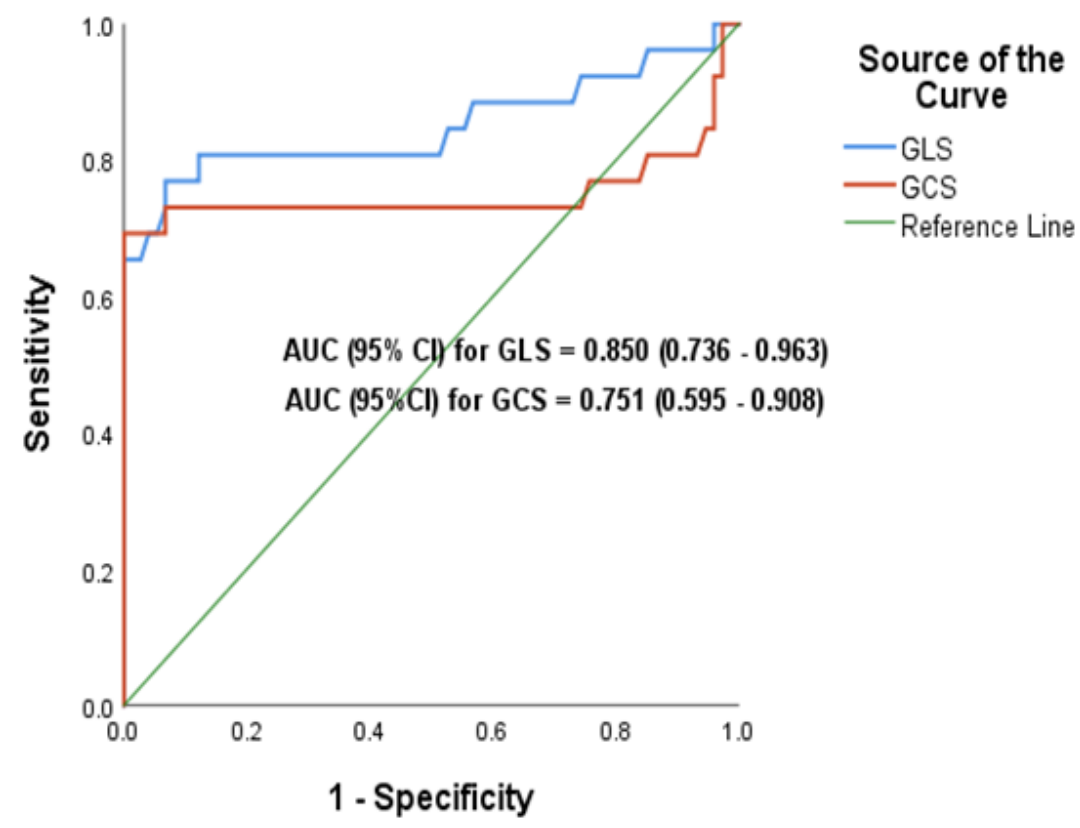

Figure 2: Receiver operating curve (ROC) analysis for the prediction of left ventricular remodeling 3 months after acute myocardial infarction. 


\section{Discussion:}

The LV undergo changes in volume, geometry, and function after STEMI, a process known as adverse post-infarction remodeling (7). The incidence of LV remodeling has decreased in the era of PCI and use of anti-remodeling medications as angiotensin-converting enzyme inhibitors and beta-blockers. Despite the improvement in management of MI, LV remodeling remains frequent after anterior MI (8).

Post infarct left ventricular remodeling increases the risk of heart failure or sudden death due to a lethal arrhythmia (9). However, there are clinical evidences that post infarct LV remodeling can be prevented or, reversed in some cases (10). Therefore, early prediction of $\mathrm{LV}$ remodeling at the time of $\mathrm{MI}$ is very essential to proper management of patients at risk of LV remodeling.

In the present study, $26 \%$ of patients developed LV remodeling after 3 months. This is consistent with $\mathrm{Na}$ et al. (11) who reported that $25.5 \%$ of successfully reperfused acute STEMI patients developed LV remodeling. Similarly, Mele et al. (12) showed that $24 \%$ of STEMI patients developed LV remodeling.

Our study revealed that there was no significant statistical difference between patients with and without remodeling regarding baseline demographic, clinical, and conventional echocardiographic data. However, we reported that patients with LV remodeling had lower LV global longitudinal
(LVGLS) and circumferential (LVGCS) strain compared to those without remodeling. This was similar to Mele et al. (12) who showed that LVGLS was significantly reduced in the remodeling group $(-11.2 \pm 2.5$ vs. $-14.8 \pm 3.2$ $\% ; \mathrm{P}=0.003)$

In the present study, multivariate analysis using the forward stepwise method showed that LVGLS was the only independent predictor of $\mathrm{LV}$ remodeling in patients with acute anterior myocardial infarction treated with primary PCI. LVGLS cutoff value of < 9.0 and LVGCS of $<-11.1$ had an important role in predicting LV remodeling. Similarly, Lacalzada et al. (13) illustrated that LVGLS can predict LV remodeling in STEMI patients. Grabka et al. (14) reported that speckle tracking-derived longitudinal strain of the left ventricular anterior wall (AGLS) supplied by the LAD is an important predictor of LV remodeling.

\section{Conclusion:}

LV remodeling occurred in $26 \%$ of patients with anterior STEMI treated by primary PCI. Patients with LV remodeling had lower LV global longitudinal and circumferential strain. LVGLS of $<-9.0$ and GCS of $<-11.1$ can predict LV remodeling in anterior STEMI. Early detection of those patients at risk of LV remodeling is important to allow for proper management and close follow up. 


\section{References}

1-Cohn JN, Ferrari R, and Sharpe N. Cardiac remodeling

- concepts and clinical implications: a consensus paper from an international forum on cardiac remodeling. $\mathbf{J}$ Am Coll Cardiol 2000; 35:569-82.

2-Azevedo P.S., Polegato B.F., Minicucci M.F., et al. Cardiac remodeling: concepts, clinical impact, pathophysiological mechanisms and pharmacologic treatment. Arquivos Brasileiros De Cardiologia 2015; (4):0.

3-Flachskampf FA, Schmid M, Rost C, et al. Cardiac imaging after myocardial infarction. European Heart Journal 2011; 32(3): 272-83.

4-Sarai M, Biswas $\mathrm{S}$, Toyama $\mathrm{H}$, et al. Ventricular remodeling following acute myocardial infarction and its correlation with discordant perfusion and fatty acid metabolism. J Nucl Med 2009; 50:1136.

5-Lang RM, Badano LP, Mor-Avi V, et al. Recommendations for cardiac chamber quantification by echocardiography in adults: an update from the American Society of Echocardiography and the European Association of Cardiovascular Imaging. European Heart Journal Cardiovascular Imaging 2015; 16(3):233-71.

6-Hung CL, Verma A, Uno H, et al. Longitudinal and Circumferential Strain Rate, Left Ventricular Remodeling, and Prognosis After Myocardial Infarction. Journal of the American College of Cardiology 2010; 56(22):1812-22.

7-Bolognese L, Neskovic AN, Parodi G, et al. Left ventricular remodeling after primary coronary angioplasty: patterns of left ventricular dilation and long-term prognostic implications. Circulation 2002; 106:2351-57.
8-Cung TT, Morel O, Cayla G, et al. Cyclosporine before PCI in patients with acute myocardial infarction. N Engl J Med 2015; 373:1021-31.

9- Verma A, Meris A, Skali H, et al. Prognostic implications of left ventricular mass and geometry following myocardial infarction: the VALIANT (Valsartan in Acute Myocardial Infarction) Echocardiographic Study. JACC: Cardiovascular Imaging 2008; 1(5): 582-91.

10-Koitabashi $\mathrm{N}$ and Kass DA. Reverse remodeling in heart failure-mechanisms and therapeutic opportunities. Nature Reviews Cardiology 2012; 9(3): 147-57.

11-Na HM, Cho GY, Lee JM, et al. Echocardiographic Predictors for Left Ventricular Remodeling after Acute ST segment Elevation Myocardial Infarction with Low Risk Group. J Cardiovasc Ultrasound 2016; 24(2):128134.

12-Mele D, Nardozza M and Chiodi E. Early speckletracking echocardiography predicts left ventricle remodeling after acute ST-segment elevation myocardial infarction. J Cardiovasc Echography 2017; 27:93-8

13-Lacalzada J, de la Rosa A, Izquierdo MM, et al. Left ventricular global longitudinal systolic strain predicts adverse remodeling and subsequent cardiac events in patients with acute myocardial infarction treated with primary percutanous coronary intervention. Int $\mathbf{J}$ Cardiovasc Imaging 2015; 3: 575-584.

14-Grabka M, Kocierz-Woźnowska M, Wybraniec M, et al. Left ventricular reverse remodeling in patients with anterior wall ST-segment elevation acute myocardial infarction treated with primary percutaneous coronary intervention. Postepy Kardiol Interwencyjnej 2018; 14(4): 373-382.

To cite this article: Al-Shimaa M. Sabry, Khaled E. El-Rabat, Ali I. Attia, Hager I. Abd El-Fatah. Left ventricular remodeling in patients with primary percutaneous coronary intervention for anterior myocardial infarction, BMFJ.2020; 37 (3):731-738. DOI: 10.21608/bmfj.2020.112400 